\title{
EIF5A2 promotes tumor metastasis and angiogenesis
}

\author{
Yan $\mathrm{Li}^{1}$, Xin-Yuan Guan ${ }^{1,2}$ \\ ${ }^{1}$ Sun Yat-sen University Cancer Center, State Key Laboratory of Oncology in South China, Collaborative Innovation Center for \\ Cancer Medicine, Guangzhou, China \\ ${ }^{2}$ Department of Clinical Oncology, The University of Hong Kong, Hong Kong, China
}

Correspondence: Xin-Yuan Guan or Yan Li

E-mail: xyguan@hkucc.hku.hk or liy6@mail.sysu.edu.cn

Received: February 09, 2014

Published online: October 15, 2014

To cite this article: Yan Li, et al. EIF5A2 promotes tumor metastasis and angiogenesis. Sci Proc 2014; 1: eXXX. doi: $10.14800 /$ sp.381.

The pathogenesis of solid tumors is a long-term process involving the accumulation of multiple genetic and epigenetic changes causing activation of oncogenes and inactivation of tumor suppressor genes. Gene amplification is one of the most common causes of oncogene activation that plays a critical role in the development and progression of various cancers because over expression of candidate oncogene(s) within the amplicon confers a selective growth advantage. Amplification of the long arm of chromosome 3, especially $3 \mathrm{q} 26$, is one of the most frequently detected genomic alterations in various cancers, such as lung cancer ${ }^{[1,}$ ${ }^{2]}$, esophageal carcinoma ${ }^{[3]}$, breast cancer ${ }^{[4]}$, ovarian cancer ${ }^{[5]}$ and nasopharyngeal carcinoma ${ }^{[6]}$, suggesting the existence of one or more important oncogenes at 3q26. To identify candidate oncogene(s) within the 3q26 amplicon, genomic DNA library of 3 q26 was generated by chromosome micro dissection and a hybrid selection strategy was applied to isolate over expressed genes from the micro dissected DNA ${ }^{[7]}$. One candidate oncogene at $3 \mathrm{q} 26$, eukaryotic translation initiation factor 5A2 (eIF5A2) was identified by this strategy. In the same time, Johansson and his colleagues also identified eIF5A2 as a phylogenetically conserved variant of eIF5A ${ }^{[8]}$. EIF5A2 shares $83 \%$ amino acid identity with EIF5A including the critical functional domain necessary for maturation by hypusine modification at lysine-50 residue ${ }^{[7,9,}$ ${ }^{10]}$. Unlike EIF5A that is universally expressed in tissues, EIF5A2 is only found in testis, brain and tumor tissues ${ }^{[8]}$.
Since eIF5A2 was firstly reported to be amplified in ovarian cancer ${ }^{[7]}$, overexpression of eIF5A2 has been reported in many other solid tumors including hepatocellular carcinoma (HCC) ${ }^{[10,11]}$, esophageal squamous cell carcinoma (ESCC) ${ }^{[12]}$, gastric cancer ${ }^{[13]}$, non-small cell lung cancer $^{[2]}$, bladder cancer ${ }^{[14,15]}$, melanoma ${ }^{[16]}$ and colorectal cancer ${ }^{[17,18]}$. Interestingly, overexpression of eIF5A2 has been closely associated with clinical stage and tumor metastasis in several cancers including ovarain cancer ${ }^{[19]}$, melanoma ${ }^{[16]}$, colorectal cancer ${ }^{[18]}$ and $\mathrm{HCC}^{[10]}$. In addition, overexpression of eIF5A2 has been also closely correlated with poorer outcome of several cancers, such as ovarian cancer ${ }^{[20]}$, bladder cancer ${ }^{[15]}$, ESCC ${ }^{[12]}$ and pancreatic cancer ${ }^{[21]}$. Metastasis consists of a series of rate limiting steps. Metastasis is the acquisition of motility by tumor cells in a process: epithelial-mesenchymal transition (EMT). Recent studies have demonstrated that eIF5A2 plays an important role in EMT through the down-regulation of epithelial markers (e.g. E-cadherin and $\beta$-catenin) and up-regulation of mesenchymal markers (e.g. fibronectin and vimentine) ${ }^{[10,12]}$. In addition, these studies find that overexpression of eIF5A 2 is able to promote the formation of stress fiber and lamellipodia via the activation of RhoA/Rac1 signaling pathway ${ }^{[10,12]}$. Molecular studies indicate that eIF5A2 is able to promote tumor metastasis by enhancing the binding of c-myc on MTA1 promoter ${ }^{[18]}$, to induce EMT via activating STAT3/ TGF- $\beta 1$ signaling ${ }^{[15]}$ and to increase MMP-2 activity ${ }^{[16]}$. 
Although 3q26 amplification is a major event for eIF5A2 over expression in ovarian cancer ${ }^{[7]}$, amplification of $3 \mathrm{q} 26$ has not been frequently detected in many other cancers, such as $\mathrm{HCC}^{[22]}$. In ESCC, the copy number gain was detected in less than $40 \%$ of ESCCs with eIF5A2 over expression ${ }^{[12]}$, suggesting that other mechanisms might contribute to the eIF5A2 over expression event. Further study finds that eIF5A2 expression can be induced by hypoxic culture condition ${ }^{[12]}$. Hypoxia is a powerful driving force to breakdown normal tissue homeostasis and rearrangement of tumor-stroma interactions in tumor invasion and metastasis ${ }^{[23]}$. Hypoxia-inducible factor-1 (HIF1) is one of the best characterized hypoxia response pathways. HIF1 $\alpha$ is one of the master regulators of tumor metastasis, which can be regulated through oxygen-dependent and -independent mechanisms in a cell specific manner ${ }^{[24]}$. Our study demonstrated that bidirectional regulation exists between EIF5A2 and HIF1 $\alpha^{[12]}$. The direct binding of EIF5A2 to the promoter region of HIF1 $\alpha$ has been verified by luciferase assay and chromatin immunoprecipitation ${ }^{[12]}$. Since HIF1 $\alpha$ also plays a very important role in tumor angiogenesis, the association of EIF5A2 over expression and angiogenesis has been studied. The results demonstrate that over expression of EIF5A2 can promote angiogenesis, and association study on clinical ESCC specimens indicates that over expression of EIF5A2 is positively correlated with HIF1 $\alpha$ and VEGF expressions ${ }^{[12]}$. In HCC, eIF5A2 has been reported to be associated with venous infiltration too ${ }^{[25]}$.

eIF5A2 has been associated with chemo resistance in breast cancer ${ }^{[26]}$, therefore, it is a potential therapeutic target for cancer treatment. shRNAs targeting eIF5A2 along or with the combination of chemotherapeutic agent docetaxel (TXT) or cisplatin (CDDP) can effectively reduce the tumor volume and tumor weight in animal models ${ }^{[12]}$. Inhibiting eIF5A2 activity with N1-guanyl-1, 7-diaminoheptane (GC7) has demonstrated to enhance the therapeutic efficacy of doxorubicin in HCC and bladder cancer cells ${ }^{[27,28]}$. As eIF5A2 plays an important role in the multi-step progression of metastasis and angiogenesis, therapeutic targeting eIF5A2 may become a new useful approach to prevent and/or control tumor metastasis and angiogenesis in cancer treatment. Taken together, recent studies have demonstrated that eIF5A2 plays critical roles in tumor cell proliferation, invasion, metastasis, angiogenesis and drug resistance. As silencing eIF5A2 expression can dramatically inhibit its oncogenic function, targeting this oncogene might shed light on the effective treatment of cancer patients with eIF5A2 over expression.

\section{References}

1. Balsara BR, Sonoda G, du Manoir S, Siegfried JM, Gabrielson E, Testa JR. Comparative genomic hybridization analysis detects frequent, often high-level, overrepresentation of DNA sequences at $3 \mathrm{q}, 5 \mathrm{p}, 7 \mathrm{p}$, and $8 \mathrm{q}$ in human non-small cell lung carcinomas. Cancer Res 1997; 57:2116-2120.

2. He LR, Zhao HY, Li BK, Liu YH, Liu MZ, Guan XY, et al. Overexpression of eIF5A-2 is an adverse prognostic marker of survival in stage I non-small cell lung cancer patients. Int J Cancer 2011; 129:143-150.

3. Kwong D, Lam A, Guan X, Law S, Tai A, Wong J, et al. Chromosomal aberrations in esophageal squamous cell carcinoma among Chinese: gain of $12 p$ predicts poor prognosis after surgery. Hum Pathol 2004; 35:309-316.

4. Forozan F, Mahlamäki EH, Monni O, Chen Y, Veldman R, Jiang $\mathrm{Y}$, et al. Comparative genomic hybridization analysis of 38 breast cancer cell lines: a basis for interpreting complementary DNA microarray data. Cancer Res 2000; 60:4519-4525.

5. Sham JS, Tang TC, Fang Y, Sun L, Qin LX, Wu QL, et al. Recurrent chromosome alterations in primary ovarian carcinoma in Chinese women. Cancer Genet Cytogenet 2002; 133:39-44.

6. Fang Y, Guan X, Guo Y, Sham J, Deng M, Liang Q, et al. Analysis of genetic alterations in primary nasopharyngeal carcinoma by comparative genomic hybridization. Genes Chromosomes Cancer 2001; 30:254-260.

7. Guan XY, Sham JS, Tang TC, Fang Y, Huo KK, Yang JM. Isolation of a novel candidate oncogene within a frequently amplified region at 3q26 in ovarian cancer. Cancer Res 2001; 61:3806-3809.

8. Jenkins ZA, Haag PG, Johansson HE. Human eIF5A2 on chromosome $3 q 25-q 27$ is a phylogenetically conserved vertebrate variant of eukaryotic translation initiation factor $5 \mathrm{~A}$ with tissue-specific expression. Genomics 2001; 71:101-109.

9. Clement PM, Henderson CA, Jenkins ZA, Smit-McBride Z, Wolff $\mathrm{EC}$, Hershey $\mathrm{JW}$, et al. Identification and characterization of eukaryotic initiation factor 5A-2. Eur J Biochem 2003; 270:4254-4263.

10. Tang DJ, Dong SS, Ma NF, Xie D, Chen L, Fu L, et al. Overexpression of eukaryotic initiation factor 5A2 enhances cell motility and promotes tumor metastasis in hepatocellular carcinoma. Hepatology 2010; 51:1255-1263.

11. Lee NP, Tsang FH, Shek FH, Mao M, Dai H, Zhang C, et al. Prognostic significance and therapeutic potential of eukaryotic translation initiation factor 5A (eIF5A) in hepatocellular carcinoma. Int J Cancer 2010; 127:968-976.

12. Li Y, Fu L, Li JB, Qin Y, Zeng TT, Zhou J, et al. Increased expression of EIF5A2, via hypoxia or gene amplification, contributes to metastasis and angiogenesis of esophageal squamous cell carcinoma. Gastroenterology 2014; 146:1701-1713.

13. Marchet A, Mocellin S, Belluco C, Ambrosi A, DeMarchi F, Mammano E, et al. Gene expression profile of primary gastric cancer: towards the prediction of lymph node status. Ann Surg Oncol 2007; 14:1058-1064.

14. Chen W, Luo JH, Hua WF, Zhou FJ, Lin MC, Kung HF, et al. Overexpression of EIF-5A2 is an independent predictor of outcome in patients of urothelial carcinoma of the bladder treated with radical cystectomy. Cancer Epidemiol Biomarkers Prev 2009; 18:400-408.

15. Wei JH, Cao JZ, Zhang D, Liao B, Zhong WM, Lu J, et al. EIF5A2 predicts outcome in localised invasive bladder cancer and 
promotes bladder cancer cell aggressiveness in vitro and in vivo. Bri J Cancer 2014; 110:1767-1777.

16. Khosravi S, Wong RP, Ardekani GS, Zhang G, Martinka M, Ong $\mathrm{CJ}$, et al. Role of EIF5A2, a downstream target of Akt, in promoting melanoma cell invasion. Bri J Cancer 2014; 110:399-408.

17. Xie D, Ma NF, Pan ZZ, Wu HX, Liu YD, Wu GQ, et al. Overexpression of EIF-5A2 is associated with metastasis of human colorectal carcinoma. Hum Pathol 2008; 39:80-86.

18. Zhu W, Cai MY, Tong ZT, Dong SS, Mai SJ, Liao YJ, et al. Overexpression of EIF5A2 promotes colorectal carcinoma cell aggressiveness by upregulating MTA1 through C-myc to induce epithelial-mesenchymaltransition. Gut 2012; 61:562-575.

19. Guan XY, Fung JM, Ma NF, Lau SH, Tai LS, Xie D, et al. Oncogenic role of eIF-5A2 in the development of ovarian cancer. Cancer Res 2004; 64:4197-4200.

20. Yang GF, Xie D, Liu JH, Luo JH, Li LJ, Hua WF, et al. Expression and amplification of eIF-5A2 in human epithelial ovarian tumors and over expression of EIF-5A2 is a new independent predictor of outcome in patients with ovarian carcinoma. Gynecol Oncol 2009; 112:314-318.

21. Wei YX, Chen G, You L, Zhao YP. Expression of eukaryotic translation initiation factor $5 \mathrm{~A} 2$ in pancreatic adenocarcinoma and its correlation with the prognosis. Zhongguo Yi Xue Ke Xue Yuan Xue Bao 2013; 35:634-638.

22. Guan XY, Fang Y, Sham JS, Kwong DL, Zhang Y, Liang Q, et al.
Recurrent chromosome alterations in hepatocellular carcinoma detected by comparative genomic hybridization. Genes Chromosomes Cancer 2000; 29:110-116.

23. Harris AL. Hypoxia--a key regulatory factor in tumour growth. Nat Rev Cancer 2002; 2:38-47.

24. Semenza GL. Targeting HIF-1 for cancer therapy. Nat Rev Cancer 2003; 3:721-732.

25. Shek FH, Fatima $S$, Lee NP. Implications of the use of eukaryotic translation initiation factor 5A (eIF5A) for prognosis and treatment of hepatocellular carcinoma. Int J Hepatol 2012; 2012:760928.

26. Liu Y, Du F, Chen W, Yao M, Lv K, Fu P. (2014). EIF5A2 is a novel chemoresistance gene in breast cancer. Breast Cancer 2014 Mar 18. [Epub ahead of print].

27. Lou B, Fan J, Wang K, Chen W, Zhou X, Zhang J, et al. N1-guanyl-1,7- diaminoheptane (GC7) enhances the therapeutic efficacy of doxorubicin by inhibiting activation of eukaryotic translation initiation factor 5A2 (eIF5A2) and preventing the epithelial-mesenchymal transition in hepatocellular carcinoma cells. Exp Cell Res 2013; 319:2708-2717.

28. Yang J, Yu H, Shen M, Wei W, Xia L, Zhao P. N1-guanyl-1,7-diaminoheptane sensitizes bladder cancer cells to doxorubicin by preventing epithelial-mesenchymal transition through inhibition of eukaryotic translation initiation factor $5 \mathrm{~A} 2$ activation. Cancer Sci 2014; 105:219-227. 